\section{Lower airway microbiota for 'biomarker' measurements of cystic fibrosis disease progression?}

\author{
Laura J Sherrard, ${ }^{1}$ Scott C Bell ${ }^{2,3,4}$
}

A series of studies in recent years have performed culture-independent methodologies, such as $16 \mathrm{~S}$ rRNA gene sequencing of bacterial DNA extracted from patient clinical samples, to infer the microbiota of different human niches and to differentiate between health and disease. Such studies of respiratory samples from people with cystic fibrosis (CF) have demonstrated that the lower airway microbiota evolves with age. Importantly, increased bacterial richness during early life progresses to a loss in bacterial community diversity and increased dominance by the common and historically recognised CF taxa such as Pseudomonas and Burkholderia. ${ }^{1-4}$ The latter features are also typical of those with the most advanced or progressive respiratory disease and are likely to be at least, in part, driven by antibiotic exposure. ${ }^{1}$ The lower airway microbiota has also been compared between disease stability and pulmonary exacerbations and changes in the relative abundance of taxa may be indicative of the onset of an exacerbation in some patients. ${ }^{5}$ Despite these important findings, it remains unclear how knowledge of the lower airway microbiota could be exploited in routine clinical practice.

Given the paucity of longitudinal culture-independent studies of the CF lower airway microbiota and associations with clinical outcomes, the paper by Acosta and colleagues published in Thorax this month is timely. ${ }^{6}$ The work provides evidence that ecological indices calculated from the lower airway microbiota could potentially be applied as 'biomarkers' to predict those who are at risk of early disease progression.

In the study, ${ }^{6} 16 \mathrm{~S}$ rRNA gene sequencing of biobanked sputum samples, collected

\footnotetext{
${ }^{1}$ School of Pharmacy, Queen's University Belfast, Belfast, UK

${ }^{2}$ Lung Bacteria Laboratory, QIMR Berghofer Medical Research Institute, Brisbane, Queensland, Australia ${ }^{3}$ Adult CF Centre, The Prince Charles Hospital, Brisbane, Queensland, Australia

${ }^{4}$ Faculty of Medicine, University of Queensland, Brisbane, Queensland, Australia
}

Correspondence to Dr Scott C Bell, Adult CF Centre, The Prince Charles Hospital, Brisbane, QLD 4032, Australia; scott.bell@health.qld.gov.au prospectively over two decades from a cohort of 104 clinically stable young adults with CF (18-22 years old), was performed and related to clinical outcomes in years following sample collection. The primary outcome was end-stage lung disease (death or lung transplantation $<25$ years) and the secondary outcomes were accelerated absolute and relative lung function decline $\left(>-3 \%\right.$ per year $\left.\mathrm{FEV}_{1}\right)$ up to 5 years after the date of sputum collection. Multiple quantitative ecological indices (eg, Shannon's and Simpson's diversity indices and Observed and Chao1 richness estimators) were calculated from the respiratory microbiota perhaps due to a lack of consensus about which are most informative. Poor clinical outcomes were seen in those with more advanced disease at study baseline and also, lower bacterial community diversity that was dominated by Pseudomonas with a relative abundance of at least 50\%. Overall, these findings corroborate with observations of prior cross-sectional studies and a longitudinal study of six patients followed for 8-9 years. ${ }^{1-4}$ However, an important caveat of the findings is that there was greater evidence of associations between the ecological indices and the primary outcome compared with the secondary outcomes, especially when evaluated as an absolute lung function decline. It is not clear why these differences may be present, as patients with increased risk of death/transplantation would also be expected to experience an enhanced loss of lung function over time.

One of the major limitations of making comparisons of ecological measures of the lower airway microbiota to clinical outcomes is that significant correlations do not imply direct nor causal relationships. 16S rRNA gene sequencing also does not reflect dynamic interactions that might occur in vivo between bacteria (and other micro-organisms), and the host which may influence the community composition and structure, immune response and ultimately, impact on disease progression. ${ }^{78}$ Furthermore, the pathogenicity within a genus might differ between species but relative low taxonomic resolution of some genera by $16 \mathrm{~S}$ rRNA gene sequencing precludes confident analysis to the species level. In this study ${ }^{6}$ bacterial communities with a higher relative abundance of Streptococcus $(\geq 25 \%)$ were associated with less evidence of disease progression indicating a possible protective role of this genus. However, there have also been reports that both the Streptococcus milleri group and group A Streptococcus are implicated in pulmonary exacerbations, a key influencer of lung disease progression in $\mathrm{CF}^{9}{ }^{10}$ Bacteria in low abundance may also influence disease pathogenesis which Acosta and colleagues highlight. ${ }^{6}$ For example, higher levels $(\geq 0.75 \%)$ of Granulicatella and Gemella were associated with less disease progression and there was some evidence of detection of Stenotrophomonas $(<1 \%)$ and early death/ lung transplantation. Granulicatella and Gemella spp. are considered commensal flora in the routine laboratory and Stenotrophomonas maltophilia has been associated with disease trajectory. ${ }^{11}$

It is well recognised that advancing lung disease in CF is strongly associated with infection with Pseudomonas aeruginosa and as a result, eradication strategies, treatment of exacerbations and chronic suppression are the standard of care. $^{12}$ Therefore, it is not surprising that the authors report an association between Pseudomonas dominance in the microbiota and early end-stage disease. ${ }^{6}$ However, it was intriguing that this correlation was not replicated with either qualitative (presence/absence), phenotypic (mucoidy) or quantitative (log-level) routine culture results. Such culture results have been identified as potential predictors of poor outcomes by others. ${ }^{13} 14$ A limitation of the study was that DNA from non-viable cells was not removed prior to sequencing. This might be important given that those with advanced disease were more likely to be prescribed inhaled antibiotics which could impact on cell death and may contribute to discrepancies between molecular and culture results. DNA from non-viable cells can be removed by the addition of propidium monoazide or alternatively, the viable bacterial community can be assessed by extended-quantitative culture. ${ }^{5} 15$ In addition, this study ${ }^{6}$ does not report $P$. aeruginosa genotypes, despite earlier studies of the 'Prairie' epidemic strain being common in the same setting, and being associated with worse outcomes such as an increased risk of death or lung transplantation. ${ }^{16}$ Furthermore, the study used frozen sputum samples, some of which were collected 20 years earlier. ${ }^{6}$ Studies have shown that delays in freezing or multiple freeze-thaw events can adversely affect the sputum microbiota, yet 
short-term duration of frozen storage does not. ${ }^{17-19}$ While the current study did not determine if long-term storage impacts on the bacterial community, quality control procedures and protocols were followed to optimise DNA preservation.

We believe that the study by Acosta and colleagues makes an important contribution to knowledge of the CF lower airway microbiota and it demonstrates how a complex bacterial community may potentially be used to identify 'at-risk' cohorts. ${ }^{6}$ In an attempt to simplify interpretation of quantitative diversity indices, patients were stratified based on predefined cut-off values which the authors identified as best discriminating between outcomes. Notably a Shannon diversity Index $<1$ was predictive of the highest risk of future negative outcomes. Novel biomarkers that identify those at an increased risk of worse outcomes could help inform clinical decision-making to limit disease progression by initiating intensification of chronic therapies, review of treatment adherence and optimising nutrition.

There are questions that remain:

1. Is it feasible to use biomarker testing of the lower airway microbiota? To be widely implemented in clinical microbiological laboratories, biomarker testing should provide rapid, accurate results that are easy to apply and importantly, cost-effective. Currently, preparation of clinical samples for $16 \mathrm{~S}$ rRNA gene sequencing is laborious and expensive and the software tools and analyses require bioinformatic expertise. Furthermore, while providing data to support the use of microbiota results for potential clinical predictions, this study also highlighted that lung function at study baseline was an independent predictor of early death or lung transplantation. ${ }^{6}$ Therefore, a larger prospective cohort of patients from different CF centres are required to assess the accuracy of using community diversity as a biomarker. Such trials are possible with the Cystic Fibrosis Microbiome-determined Antibiotic Therapy Trial in Exacerbations: Results Stratified (CFMATTERS) trial currently underway and comparing the use of microbiota-directed antibiotic treatment with standard therapy for pulmonary exacerbations (https:// www.cfmatters.eu).

2. When should biomarker testing of the lower airway microbiota begin? The age range of patients with CF included in this study corresponds to those transitioning from paediatric to adult care, which is a significant milestone that re- quires careful planning. ${ }^{6} 20$ Therefore, this might represent the ideal opportunity to perform biomarker testing that could inform a personalised treatment strategy of young adults with CF at a time when clinical decline is frequently observed as the individual is transitioning to a more independent lifestyle.

3. Does the respiratory sample type matter? Sputum was used to infer the lower airway microbiota in this study. ${ }^{6}$ Adolescents transitioning from paediatric to adult CF care are becoming progressively healthier (improved lung function and nutritional status) ${ }^{21}$ and the use of therapies that enhance CFTR function ${ }^{22} 23$ could mean that, in the future, collection of expectorated sputum might be exceptional rather than the norm. Alternative clinical samples such as oropharyngeal or cough swabs may not represent the lower airway microbiota. For example, the recent CF-Sputum Induction Trial (CF-SpIT) demonstrated that the intrapatient microbiota of sputum is similar to bronchoalveolar lavage but different to cough swabs. ${ }^{24}$ Therefore, how generalisable the results of the study are to other sample types requires investigation.

In conclusion, Acosta and colleagues provide novel data on a topic that is of great interest to researchers and healthcare professionals alike. The use of longterm outcomes is a strength and the study has begun to address the knowledge gap of how the results of culture-independent analyses could potentially inform clinical care. Future studies of other cohorts (eg, younger cohorts, older cohorts and endpoints considered beyond 25 years of age) using the same techniques and parameters to attempt to replicate the key findings will be important to convince hospital laboratories and clinicians that lower airway microbiota 'biomarkers' are ready for implementation.

Contributors LJS and SCB cowrote the editorial with input into the concept, writing and final proof reading.

Funding This study was funded by Health Innovation, Investment and Research Office (grant no: 50007), National Health and Medical Research Council (grant no: 1102494), The Prince Charles Foundation (grant no: ER2015-17).

Competing interests None declared.

Patient consent Not required.

Provenance and peer review Commissioned; externally peer reviewed.

(C) Author(s) (or their employer(s)) 2018. No commercial re-use. See rights and permissions. Published by BMJ.

Check for updates
To cite Sherrard LJ, Bell SC. Thorax

2018;73:1001-1003.

Accepted 23 July 2018

Published Online First 22 August 2018

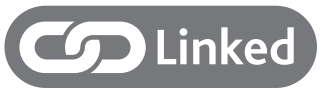

- http://dx.doi.org/10.1136/thoraxjnl-2018-211510

Thorax 2018;73:1001-1003.

doi:10.1136/thoraxjnl-2018-212165

\section{REFERENCES}

1 Zhao J, Schloss PD, Kalikin LM, et al. Decade-long bacterial community dynamics in cystic fibrosis airways. Proc Natl Acad Sci U S A 2012;109:5809-14.

2 Zemanick ET, Wagner BD, Robertson CE, et al. Airway microbiota across age and disease spectrum in cystic fibrosis. Eur Respir J 2017;50:1700832.

3 Coburn B, Wang PW, Diaz Caballero J, et al. Lung microbiota across age and disease stage in cystic fibrosis. Sci Rep 2015:5:10241.

4 Klepac-Ceraj V, Lemon KP, Martin TR, et al. Relationship between cystic fibrosis respiratory tract bacterial communities and age, genotype, antibiotics and Pseudomonas aeruginosa. Environ Microbiol 2010;12:1293-303.

5 Cuthbertson L, Rogers GB, Walker AW, et al. Respiratory microbiota resistance and resilience to pulmonary exacerbation and subsequent antimicrobial intervention. Isme J 2016;10:1081-91.

6 Acosta N, Heirali A, Somayaji R, et al. Sputum microbiota is predictive of long-term clinical outcomes in young adults with cystic fibrosis. Thorax 2018.

7 Mirković B, Murray MA, Lavelle GM, et al. The role of short-chain fatty acids, produced by anaerobic bacteria, in the cystic fibrosis airway. Am J Respir Crit Care Med 2015:192:1314-24.

8 Sherrard LJ, McGrath SJ, Mcllreavey L, et al. Production of extended-spectrum $\beta$-lactamases and the potential indirect pathogenic role of Prevotella isolates from the cystic fibrosis respiratory microbiota. Int J Antimicrob Agents 2016;47:140-5.

9 Parkins MD, Sibley CD, Surette MG, et al. The Streptococcus milleri group--an unrecognized cause of disease in cystic fibrosis: a case series and literature review. Pediatr Pulmonol 2008;43:490-7.

10 Skolnik K, Nguyen A, Somayaji R, et al. Clinical implications and characterization of Group A Streptoccoccus infections in adults with cystic fibrosis. BMC Pulm Med 2015;15:161.

11 Waters V, Atenafu EG, Lu A, et al. Chronic Stenotrophomonas maltophilia infection and mortality or lung transplantation in cystic fibrosis patients. J Cyst Fibros 2013; 12:482-6.

12 Döring G, Flume P, Heijerman H, et al. Treatment of lung infection in patients with cystic fibrosis: current and future strategies. J Cyst Fibros 2012;11:461-79.

13 Mayer-Hamblett N, Rosenfeld M, Gibson $\mathrm{RL}$, et al. Pseudomonas aeruginosa in vitro phenotypes distinguish cystic fibrosis infection stages and outcomes. Am J Respir Crit Care Med 2014;190:289-97.

14 Szczesniak RD, Li D, Su W, et al. Phenotypes of rapid cystic fibrosis lung disease progression during adolescence and young adulthood. Am J Respir Crit Care Med 2017;196:471-8.

15 Tunney MM, Field TR, Moriarty TF, et al. Detection of anaerobic bacteria in high numbers in sputum from patients with cystic fibrosis. Am J Respir Crit Care Med 2008;177:995-1001.

16 Somayaji R, Lam JC, Surette MG, et al. Long-term clinical outcomes of 'Prairie Epidemic Strain' Pseudomonas aeruginosa infection in adults with cystic fibrosis. Thorax 2017;72:333-9. 
17 Cuthbertson L, Rogers GB, Walker AW, et al. Time between collection and storage significantly influences bacterial sequence composition in sputum samples from cystic fibrosis respiratory infections. J Clin Microbiol 2014;52:3011-6.

18 Cuthbertson L, Rogers GB, Walker AW, et al. Implications of multiple freeze-thawing on respiratory samples for culture-independent analyses. J Cyst Fibros 2015;14:464-7.

19 Zhao J, Li J, Schloss PD, et al. Effect of sample storage conditions on culture-independent bacterial community measures in cystic fibrosis sputum specimens. J Clin Microbiol 2011:49:3717-8.

20 Towns SJ, Bell SC. Transition of adolescents with cystic fibrosis from paediatric to adult care. Clin Respir J 2011;5:64-75.

21 Ramsay KA, Sandhu H, Geake JB, et al. The changing prevalence of pulmonary infection in adults with cystic fibrosis: A longitudinal analysis. J Cyst Fibros 2017:16:70-7.

22 Ramsey BW, Davies J, McElvaney NG, et al. A CFTR potentiator in patients with cystic fibrosis and the G551D mutation. N Engl J Med 2011;365:1663-72.
23 Heltshe SL, Rowe SM, Skalland M, et al. Ivacaftor-treated patients with cystic fibrosis derive long-term benefit despite no short-term clinical improvement. Am J Respir Crit Care Med 2018;197:1483-6.

24 Ronchetti K, Tame JD, Paisey C, et al. The CFSputum Induction Trial (CF-SpIT) to assess lower airway bacterial sampling in young children with cystic fibrosis: a prospective internally controlled interventional trial. Lancet Respir Med 2018;6:461-71. 